\title{
Consumo de suplementos por alunos de academias de ginástica em São Paulo
}

\section{Supplement consumption among fitness center users in São Paulo, Brazil}

Raquel Franzini PEREIRA ${ }^{1}$

Franco Maria LAJOLO2

Marcia Daskal HIRSCHBRUCH ${ }^{3}$

\section{RE S U M O}

O aumento do número de academias de ginástica na cidade de São Paulo, em conjunto com o aumento da oferta de diferentes suplementos no mercado, despertou interesse para o estudo do consumo de suplementos entre seus alunos. 0 uso de suplementos pelo público em geral não é bem quantificado e pouca informação sobre este assunto está publicada na literatura. 0 seu aparecimento no mercado tem sido mais rápido do que a elaboração de regulamentações e a realização de pesquisas científicas que comprovem seus efeitos na saúde dos consumidores e determinem a segurança de seu uso a longo prazo. Em uma amostra de 309 freqüentadores de sete academias de ginástica de São Paulo em 1999, 74 (23,9\% ) consumiam algum tipo de suplemento, dos quais $77,0 \%$ eram do sexo masculino e $23,0 \%$ do sexo feminino. Os suplementos mais consumidos foram aminoácidos ou outros concentrados protéicos $(38,9 \%)$ e o consumo maior foi o diário $(90,3 \%)$. A correlação entre gasto com suplemento e renda individual foi de $27,5 \% \quad(p=0,0483 ; n=52)$, sendo o gasto com suplementos maior entre homens do que entre mulheres; a correlação com renda familiar foi de $36,1 \%$ $(p=0,0137 ; n=46)$ e com Índice de Massa Corporal foi de 17,1\% ( $p=0,1564 ; n=70)$. Conclui-se que 0 uso de suplementos é significante no grupo analisado, ficando clara a necessidade de novos estudos sobre o consumo desses produtos e seus efeitos, enfocando aspectos de educação nutricional do consumidor de suplementos para aumentar o nível de informação sobre os mesmos e garantir segurança na sua utilização.

Termos de indexação: nutrição esportiva, aminoácidos, suplementos dietéticos, academias de ginástica, educação nutricional.

\footnotetext{
1 M estranda, Curso de Nutrição Humana, Universidade de São Paulo.

2 Faculdade de Ciências Farmaceuticas, Universidade de São Paulo.

3 Mestranda, Universidade Federal de São Paulo/Escola Paulista de Medicina. Rua Pará, 76/92, 01243-020, Higienópolis, São Paulo, SP, Brasil. Correspondência para/Correspondenceto: M.D. HIRSCHBRUCH. E-mail: recomendo@hotmail.com
} 


\section{A B S T R A C T}

The increase in the number of fitness centers in the city of São Paulo, together with the increase in the offering of different supplements, has raised interest in the study of the consumption of supplements among fitness center users. The consumption of supplements by the general public has not been well quantified yet, and little information has been published about this subject. The arrival of new supplements in the market place has been faster than the creation of regulations and the accomplishment of researches proving their effects and determining the safety for their long-term use. In a sample of 309 users from seven fitness centers in São Paulo in 1999, 74 (23.9\%) used at least one type of supplement, $77,0 \%$ of whom were male and $23,0 \%$ were female. The most consumed supplements were amino acids or other proteic products $(38.9 \%)$, and the daily intake was the most mentioned consumption frequency $(90.3 \%)$. The correlation between money expenditure with supplement and individual income was $27.5 \%(p=0.0483 ; n=52)$, and the expenses were greater among men; the correlation with family income was $36.1 \%(p=0.0137 ; n=46)$ and with Body Mass Index it was to $17.1 \%(p=0.1564 ; n=70)$. The conclusion was that the consumption of supplements is significant in the evaluated group, showing the need for more studies about the consumption of these products and their effects, focusing on nutrition education approaches in order to increase the level of information about them and to guarantee the safety of their use.

Index terms: sports nutrition, amino acids, dietary supplements, fitness centers, nutrition education.

\section{N T R O D U Ç Ã O}

Freqüentadores de academias de ginástica são, em geral, indivíduos com alto nível de escolaridade, com motivação e recursos para a prática de atividades físicas e para uma alimentação saudável e com acesso a informações so bre nutrição e atividade física. A tualmente, as evidências científicas incentivam a prática de exercícios físicos e a adoção de uma alimentação equilibrada.

Sabe-se que o número de academias tem aumentado nos últimos anos. Conforme relatos da imprensa leiga, cerca de 3 milhões de pessoas praticavam exercícios físicos na cidade de São Paulo em 1998 e o total de academias de ginástica na cidade passou de 600 a 3 mil (excluindo as academias dentro de grandes empresas) na última década ${ }^{1}$.

O aumento do número de academias na cidade de São Paulo, em conjunto com o aumento da of erta de diferentes suplementos no mercado, despertou interesse para o estudo do consumo de suplementos deste grupo, com o objetivo de identificar melhor este uso. Isto é importante, pois pode fornecer informações úteis para se determinar futuramente a segurança e eficiência do consumo de suplementos, bem como para embasar a aplicação de programas de educação nutricional.

De acordo com Eliason et al. (1997)2, os suplementos podem ser definidos como produtos feitos de vitaminas, minerais, produtos herbais, extratos de tecidos, proteínas e aminoácidos e outros produtos, consumidos com o objetivo de melhorar a saúde e prevenir doenças. A pesar de a American Dietetic Association (ADA) afirmar que a melhor estratégia nutricional para a promoção da saúde e redução do risco de doença crônica é obter os nutrientes adequados através de uma alimentação variada, ela ainda considera apropriado o uso de suplementos de vitaminas e minerais quando evidências científicas bem aceitas e revisadas demonstram segurança e eficiência em seu consumo ${ }^{3}$.

Cada dia mais as pessoas acreditam que a saúde seja comprável, sendo a venda de suplementos dietéticos crescente; nos Estados Unidos este mercado já ultrapassou a cifra de 6,5 
bilhões de dólares em 1996 (englobando principalmente vitaminas e minerais), segundo Kurtzweil (1998) ${ }^{4}$. No entanto, Barret (1997) $)^{5}$ alerta para o fato de que milhares de "suplementos" são comercializados com a falsa promessa de aumentar a energia, aliviar o estresse, aumentar a performance atlética e prevenir ou tratar inúmeros problemas de saúde, pois muitos destes produtos não têm o efeito prometido no rótulo comprovado por estudos científicos, como demonstram Grunewald \& Bailey $(1993)^{6}$. Por exemplo, os fat burners não têm seu efeito sugerido de queimar mais gorduras realmente comprovado cientificamente 7 . Portanto, especialistas em nutrição precisam ter um papel ativo, ajudando consumidores a reconhecer as informações erradas e prejudiciais ${ }^{8}$.

Pouca informação sobre o consumo de suplementos pelo público em geral está publicada na literatura científica. Pode-se ainda haver falta de conhecimento por parte dos profissionais da saúde sobre a popularidade dos suplementos e seus efeitos potenciais e colaterais ${ }^{2,9}$.

Kubena \& M cMurray (1996) $)^{10}$ acreditam que haja a necessidade de pesquisas sobre as conseqüências do uso de suplementos com múltiplos nutrientes, especialmente 0 efeito de alguns nutrientes na função imune, pois o interesse do consumidor na suplementação para aumentar esta função pode gerar danos no futuro.

A ADA apóia a pesquisa sobre alimentos funcionais, a fim de se desvendar melhor seus componentes ativos, benefícios e riscos para a saúde, aceitando que eles têm um efeito potencialmente benéfico na saúde quando consumidos regularmente e em níveis eficientes como parte de uma dieta variada ${ }^{3}$. Os nutricionistas deveriam encorajar o consumo de uma dieta variada, com alimentos densos em nutrientes, para promover 0 equilíbrio adequado entre todos os nutrientes. Até o momento, o melhor conselho dietético para aumentar a função imune em pessoas saudáveis parece ser a variedade, o equilíbrio e a moderação alimentar ${ }^{10}$. Afinal, os alimentos não podem ser mais avaliados apenas em termos de macronutrientes e micronutrientes, já que contêm outros componentes ativos ${ }^{3}$.

Os atuais suplementos dietéticos já são inúmeros, mas as controvérsias no meio científico sobre seus possíveis efeitos, riscos e benefícios, confundiram muito os consumidores. As próprias definições de suplementos são demasiadamente amplas e não contribuem para o esclarecimento de suas funções para gerarem mais confusão para o público leigo.

Devido a esse aumento de informação sobre saúde, alimentação e suplementos, maiores esforços devem ser concentrados na educação nutricional do público em geral. A educação nutricional, assim como o consumo de alimentos e de suplementos, tende a ser uma área crescente que deve despertar ainda mais interesse e reafirmar sua importância nos próximos anos.

Nesse sentido, objetivou-se com este trabalho avaliar o consumo de suplementos entre freqüentadores de academias de ginástica em São Paulo, através da análise do número de consumidores, dos tipos de produtos ingeridos, das fontes de indicação, das atividades físicas praticadas, da freqüência de consumo e de outras informações, contribuindo assim para futuros estudos e para programas de educação nutricional.

\section{CASUÍSTICA E MÉTODOS}

Foi realizado um estudo transversal com análise descritiva, com base em dados primários obtidos para esta pesquisa.

A população de estudo foi composta por frequentadores de sete diferentes academias de ginástica, cujo total de indivíduos matriculados no mês da pesquisa variou entre 126 e 2500 . Os estabelecimentos ofereciam atividades físicas diferentes, direcionadas a indivíduos de faixas etárias diversas. Os critérios utilizados para a escolha das academias foram: estarem localizadas 
em bairros centrais da cidade ou próximos a grandes centros comerciais, oferecerem diversas atividades físicas, apresentarem diferentes tamanhos e não estarem restritas a uma faixa etária específica. Considerou-se freqüentadores aqueles que vão pelo menos duas vezes por semana, por 45 minutos ou mais por vez, para a prática de exercícios físicos.

Para a coleta de dados, um formulário com questões sobre consumo de suplemento (tipo de suplemento, freqüência de consumo, indicação de produto, entre outras informações) foi aplicado à amostra selecionada. Além de perguntas sobre consumo de suplementos, o formulário continha outras questões pertinentes à dissertação de mestrado para a qual este estudo foi realizado.

Os alunos foram abordados de forma aleatória na entrada das academias, em diferentes horários do dia e em diferentes dias da semana. Aqueles que preencheram voluntariamente 0 formulário de pesquisa foram chamados de participantes neste estudo, cientes de estarem participando voluntariamente e de não haver nenhuma conseqüência pela sua não-participação.

A faixa etária dos indivíduos para o preenchimento dos formulários foi determinada entre 18 e 38 anos, por representar a maioria dos alunos, de acordo com a distribuição por idade e sexo fornecida pelas academias. Os participantes assinaram um termo de consentimento para a autorização da utilização dos dados de forma sigilosa com finalidade de pesquisa científica.

Os dados foram avaliados através de correlações de Pearson e análises de variância. 0 programa estatístico utilizado foi o Statistical Analysis System (SAS), versão 6.11.

\section{RESU LT A D O S}

Obtiveram-se 309 formulários preenchidos no total. Observou-se que 216 indivíduos $(69,9 \%$ ) tinham nível de escolaridade superior (completo ou não), moravam em 79 bairros diferentes da cidade e tinham renda mensal individual de $\mathrm{R} \$ 2.054,00 \pm 2.154,9$ (mediana de $\mathrm{R} \$ 1.300,00$, coeficiente de variação $=105,0 \%$ ). A maioria ( $n=223 ; 72,2 \%$ ) possuía peso normal de acordo com o Índice de M assa Corporal (IMC), e apenas 63 freqüentadores $(20,4 \%$ ) apresentavam excesso de peso.

Entre os 74 (23,9\%) participantes que consomem pelo menos um suplemento, 57 $(77,0 \%)$ são do sexo masculino e $17(23,0 \%)$ do sexo feminino. Foi efetuada a distribuição do consumo de suplementos em relação ao número de participantes e tipo de produto de acordo com ingredientes (Tabelas 1 e 2 , respectivamente). 0 grupo de suplementos mais mencionado foi o de aminoácidos ou produtos protéicos, coerente com o fato de 132 freqüentadores (42,7\% ) praticarem atividades físicas com o objetivo de aumento de massa muscular.

Entre as fontes de prescrição ou recomendação de suplementos, a mais citada foi a do grupo dos instrutores, professores e treinadores de atividades físicas ( $n=28 / 31 / 8 \%$ ) (Tabela 3). Foi realizada também a distribuição dos participantes segundo atividade física praticada (Tabela 4).

Tabela 1. Distribuição do número e percentagem de participantes segundo consumo de suplemento.

\begin{tabular}{lcc}
\hline Consumo de suplementos & $\mathrm{n}$ & $\%$ \\
\hline Consumo de 1 suplemento & $74 *$ & 23,9 \\
Consumo de 2 suplementos & 27 & 8,7 \\
Consumo de 3 suplementos & 12 & 3,9 \\
Consumo de 4 suplementos & 5 & 1,6 \\
Não consomem suplementos & 235 & 76,1 \\
\hline Total & 353 & 100,0 \\
\hline
\end{tabular}

\footnotetext{
(*) Sendo 57 (77\%) homens e $17(23 \%)$ mulheres.
} 
Tabela 2. Distribuição do número e percentagem de suplementos mencionados segundo tipo de produto.

\begin{tabular}{|c|c|c|}
\hline Tipo de produto & $\mathrm{n}$ & $\%$ \\
\hline Aminoácidos ou outros concentrados protéicos & 42 & 38,9 \\
\hline Vitaminas ou complexos vitamínicos & 16 & 14,8 \\
\hline Carboidratos ${ }^{\mathrm{a}}$ & 12 & 11,1 \\
\hline Creatina & 11 & 10,2 \\
\hline M inerais & 1 & 0,9 \\
\hline Fat Burner ${ }^{\mathbf{b}}$ & 1 & 0,9 \\
\hline Bebida isotônica & 1 & 0,9 \\
\hline Outros ${ }^{c}$ & 24 & 22,2 \\
\hline Total & 108 & 100,0 \\
\hline
\end{tabular}

(a) Frutose, maltodextrina, mel e outros; (b) Com objetivo de queima de gordura; (c) 22 diferentes produtos foram citados.

Tabela 3. Distribuição do número e percentagem de relato de consumo de suplemento segundo fonte de indicação.

\begin{tabular}{lcc}
\hline Fonte de indicação & $\mathrm{n}$ & $\%$ \\
\hline Instrutor, treinador, professor de atividade física & 28 & 31,1 \\
Amigos & 14 & 15,6 \\
Próprio indivíduo & 14 & 15,6 \\
Nutricionista & 10 & 11,1 \\
Médico & 9 & 10,0 \\
Vendedor de loja de suplementos & 4 & 4,4 \\
Familiares & 3 & 3,3 \\
Academia & 2 & 2,2 \\
Outros & 6 & 6,7 \\
\hline Total & 90 & 100,0 \\
\hline
\end{tabular}

Tabela 4. Distribuição do número e percentagem de participantes segundo atividade física praticada.

\begin{tabular}{lcc}
\hline Atividade física praticada & $\mathrm{n}$ & $\%$ \\
\hline Conjunto de diferentes atividades $^{\mathbf{a}}$ & 135 & 43,7 \\
Exercícios anaeróbicos $^{\mathbf{b}}$ & 84 & 27,2 \\
Exercícios aeróbicos $^{\mathbf{c}}$ & 44 & 14,2 \\
Artes marciais $^{\mathbf{1}}$ & 25 & 8,1 \\
Esportes coletivos $^{\mathbf{e}}$ & 12 & 3,9 \\
Não respondeu & 9 & 2,9 \\
\hline Total & 309 & 100,0 \\
\hline
\end{tabular}

(a) Pelo menos 2 atividades de tipos diferentes de exercícios físicos; ${ }^{\text {(b) }}$ M usculação, ginástica localizada e outros; ${ }^{\text {(c) }}$ Caminhada, corrida e outros; (d) Caratê, judo e outros; (e) Futebol, basquete e outros.

A distribuição da freqüência de consumo de suplementos mostra que $102(90,3 \%)$ das menções de uso referiram consumo diário; apenas $8(7,1 \%)$ relataram consumo semanal; $2(1,8 \%)$, quinzenal e $1(0,9 \%)$ citou consumo raro.
Em relação a gastos com suplementos, a média mensal foi de $\mathrm{R} \$ 56,90 \pm 48,0$ (coeficiente de variação $=84,3 \%$ ), sendo o gasto médio mensal de $R \$ 61,70 \pm 51,9$ (coeficiente de variação $=84,1 \%$ ) para homens e de $R \$ 41,70 \pm 29,9$ 
(coeficiente de variação $=71,1 \%$ ) para mulheres.

Encontrou-se uma correlação positiva de $27,5 \%$ entre renda individual e gasto com suplementos $(p=0,0483 ; n=52)$, de $17,1 \%$ entre IMC e gasto com suplemento $(p=0,1564 ; n=70)$ e de $36,1 \%$ entre renda familiar e gasto com suplementos ( $p=0,0137 ; n=46)$.

\section{I S C U S S Ã O}

É importante ressaltar que $23,9 \%$ dos participantes do estudo consumiam algum tipo de suplemento, não necessariamente vitaminas e minerais.

Os dados encontrados em estudos sobre suplementos variam. Em pesquisa feita por Rocha \& Pereira (1998) ${ }^{11}$ em 16 academias no Estado do Rio de Janeiro, com 160 entrevistados no total, 51 indivíduos (32,0\% ) faziam uso de algum tipo de suplemento. Nos EUA, o consumo de suplementos de vitaminas e minerais pela população em geral é de $30,0 \%$ a $60,0 \%{ }^{12-14}$. Sobal \& M arquart (1994) $)^{15}$ obtiveram entre atletas de colegial nos EUA a prevalência de consumo de $38,0 \%$; já na Coréia, segundo $\operatorname{Kim} \&$ Keen $(1999)^{16}$, esta foi de $35,8 \%$, superando em muitos casos o nível de segurança de ingestão de acordo com as recomendações. Em estudo feito por Krumbach et al. (1999) ${ }^{17}$ com atletas universitários a prevalência foi de $56,7 \%$.

Neste trabalho, observou-se mais homens $(75,7 \%)$ do que mulheres $(24,3 \%)$ entre os usuários de suplementos. Em outro estudo brasileiro, feito por Rocha \& Pereira (1998) ${ }^{11}$, a maioria dos usuários de suplementos tinha de 20 a 30 anos de idade, sendo $35(69,0 \%)$ do sexo masculino e $16(31,0 \%)$ do sexo feminino. De acordo com uma pesquisa americana, no entanto, $37,2 \%$ das mulheres e $23,9 \%$ dos homens entre 20 e 29 anos tomavam suplementos de vitaminas e/ou minerais ${ }^{18}$. Além disso, entre atletas universitários, 55,3\% dos homens e 59,3\% das mulheres consumiam suplementos de vitaminas e/ou minerais, segundo estudo de Krumbach et al. (1999)17.

Entre os participantes deste estudo, o uso diário de suplementos era mais comum $(90,3 \%$ dos relatos de consumo), o que provavelmente se deve ao fato de os produtos geralmente sugerirem esta freqüência de consumo. Contudo, no estudo feito por Krumbach et al. (1999) ${ }^{17}$ apenas $29,8 \%$ dos consumidores de suplementos os ingeriam cinco vezes ou mais por semana.

Os gastos com suplementos foram maiores entre homens do que entre mulheres, coerentes com a tendência de os alunos de academias de ginástica do sexo masculino se interessarem muito pelo aumento de massa muscular e conseqüentemente recorrerem aos inúmeros suplementos existentes no mercado com este objetivo.

A fonte mais utilizada de recomendação de suplementos foi a de instrutores e professores (31,1\% das fontes de indicação), seguida de amigos $(15,6 \%)$, auto-indicação $(15,6 \%)$, nutricionista $(11,1 \%$ ) e médico $(10,0 \%)$. Contudo, é importante notar que em alguns casos professores e instrutores são vendedores destes suplementos e não recebem formação científica adequada para ter conhecimento suficiente sobre os efeitos dos mesmos. Já em estudo feito por Krumbach et al. (1999) ${ }^{17}$, a fonte mais utilizada para indicação do suplemento foi o próprio consumidor $(40,6 \%)$, seguida do nutricionista $(32,1 \%)$, familiares e amigos $(31,1 \%)$, treinadores $(23,6 \%)$ e médico ou farmacêutico $(12,2 \%)$. Segundo trabalho realizado por Rocha $\&$ Pereira $(1998)^{11}$, 32 alunos (63,0\% da amostra) receberam alguma orientação para o consumo de suplemento(s), dos quais $13(41,0 \%$ ) foram orientados por profissionais de saúde (9 por médicos e 4 por nutricionistas) e os demais 19 (59,0\% ) através de "instrutores", " amigos", "leitura sobre o assunto", entre outras fontes.

A grande procura por suplementos com 0 objetivo de aumento de massa muscular reflete 0 desejo desta população de obter este resultado, 
pois foi o motivo mais citado para a prática de atividades físicas. Contudo, em pesquisa feita por Sobal \& Marquart (1994) ${ }^{19}$, atletas consumiam suplementos com o objetivo de aumentar a performance atlética, o que não depende sempre do aumento de massa muscular.

O grupo de suplementos mais citado entre os usuários brasileiros do estudo de Rocha $\&$ Pereira (1998) ${ }^{11}$ foi o de produtos cuja composição não foi indentificada ou não se enquadrava em outro grupo, sendo citados como "energizantes", "estimulantes", entre outras denominações, seguido dos produtos com composição predominante em aminoácidos e proteínas. M as quando se perguntou ao grupo de usuários se conheciam a finalidade do suplemento por eles consumido, 1 (2\% ) não respondeu, 7 (14\% ) responderam que não conheciam e 43 (84\% ) informaram conhecê-la, dos quais apenas dois indivíduos souberam dizer com exatidão qual era a finalidade do produto. Não é raro encontrar freqüentadores de academias consumindo suplementos sem conhecer seu ingrediente principal, sua função ou por quais mecanismos este produto irá alcançar os resultados prometidos no rótulo.

Alguns indivíduos relatam que, além do efeito desejado, obtiveram também com o consumo de suplementos outros benefícios como "mais disposição e resistência" e "mais saúde e confiança em si mesmos"; no entanto, outros afirmaram que tiveram "problemas renais e hepáticos", "diminuição do desempenho sexual", " tonteira, enjôos, irritação, insônia e acne", entre outros distúrbios ${ }^{11}$.

O aparecimento dos suplementos no mercado tem sido aumentado e é emergente a necessidade de pesquisas científicas para comprovar seus efeitos e determinar a segurança de seu uso a longo prazo. A elaboração de regulamentações sobre 0 assunto, facilitaria a atuação dos profissionais de saúde e a educação do público em geral sobre o uso seguro e eficiente desses produtos.

\section{O N C L U S Ã O}

O consumo de suplementos foi significante entre os frequentadores de academias de ginástica em São Paulo e suficiente para despertar interesse de estudos mais extensos sobre este consumo, incluindo, entre outros temas, os efeitos dos suplementos e os meios para auxiliar o consumidor a fazer uso correto dos mesmos.

O usuário ainda se encontra mal informado sobre o conteúdo dos suplementos e as conseqüências de sua ingestão. M uitos desses produtos geram grande controvérsia sobre seus efeitos, principalmente a longo prazo. Adicionalmente, sua venda e sua recomendação são feitas por profissionais não especializados no assunto. Portanto, o consumo de suplementos de maneira inadvertida pode vir a representar um problema de saúde pública.

A falta de estudos suficientes e conclusivos sobre suplementos dietéticos deve-se não só ao fato de ser um assunto recente, mas também ao constante aparecimento de novos produtos no mercado. Assim, maiores esclarecimentos a respeito de seus efeitos na saúde são necessários para se evitar um problema de saúde pública, já que o consumo desse tipo de produto é significante. Além disso, programas de educação alimentar em conjunto com esclarecimentos sobre os efeitos do uso de suplementos são necessários para uma ingestão adequada de nutrientes, garantindo a saúde e prevenindo doenças.

\section{A G RADECIM EN TO}

À CAPES pela bolsa de pesquisa fornecida para a realização deste estudo.

\section{RE FERÊ N C I A S}

1. França V. Exercícios à moda paulistana. Veja São Paulo 1998; 25:12-20.

2. Eliason BC, Kruger J, Mark, D, Rasmann DN. Dietary supplement users: demographics, product use, and 
medical system interaction. Board Famly Practce 1997; 10:265-71.

3. Position of the American Dietetic Association: functional foods. J Am Diet Assoc 1999, 99:1278-85.

4. Kurtzweil P. An FDA guide to dietary supplements. FDA Consumer 1998; 32(50):28-35.

5. Barret S. Consumer health: a guide to intelligent decisions. 6th. Madison: Brown \& Benchmark Publishers; 1997.

6. Grunewald KK, Bailey RS. Commercially marketed supplements for bodybuilding athletes. Sports Med 1993; 15:90-103.

7. Cowart VS. Dietary supplements: alternatives to anabolic steroids? Physician Sportsmed 1992; 20:189-98.

8. Position of the American Dietetic Association: vitamin and mineral supplementation. Am Diet Assoc 1996; 96:73-87.

9. Philen RM , Ortiz DI, Auerbach SB, Falk H. Survey of advertising for nutritional supplements in health and bodybuilding magazines. J Am Diet Assoc 1992; 268:1008-11.

10. Kubena KS, McMurray D. Nutrition and the immune system: a review of nutrient-nutrient interactions. J Am Diet Assoc 1996; 96:1156-64.

11. Rocha LP, Pereira MVL. Consumo de suplementos nutricionais por praticantes de exercícios físicos em academias. Rev Nutr 1998, 11(1):76-82.
12. Koplan JP, Annest JL, Layde PM , Rubin GL. Nutrient intake and supplementation in the United States (NHANE II). Am J Public Health 1986; 76:287-89.

13. Kim IS, Williamson DF, Byers T, Koplan JP. Vitamin and mineral supplement use and mortality in a U.S. cohort. Am J Public Health 1993: 83:546-50.

14. Eldridge AL, Sheehan ET. Food supplement use and related beliefs: survey of community college students. J Nutr Educ 1994; 26:259-65.

15. Sobal J, Marquart LF. Vitamin/mineral supplement use among high school athletes. Adolescence 1994; 29:835-43.

16. Kim SH, Keen CL. Patterns of Vitamin/M ineral supplement usage by adolescents attending athletic high schools in Korea. Int J Sports Nutr 1999: 9:391-405.

17. Krumbach CJ, Ellis DR, Driskell JA. A report of vitamin and mineral supplement use among university athletes in a division I institution. Int J Sport Nutr 1999: 9:416-25.

18. Federation of american societies for experimental biology. Third Report on Nutrition Monitoring in the United States. Washington DC: U.S. Government Printing Office; 1995. v.1

19. Sobal J, Marquart LF. Vitamin/mineral supplement use among athtletes: a review of the literature. Int J Sports Nutr 1994; 4:320-34.

Recebido para publicação em 19 de setembro de 2001 e aceito em 11 de setembro de 2002. 